\title{
Diffuse gliomas in an adolescent with multiple enchondromatosis (Ollier's disease)
}

\author{
K. HORI ${ }^{1}$, A. MATSUMINE ${ }^{1}$, R. NIIMI ${ }^{1}$, M. MAEDA $^{2}$, K. UCHIDA ${ }^{3}$, T. NAKAMURA ${ }^{1}$ and A. SUDO ${ }^{1}$ \\ Departments of ${ }^{1}$ Orthopaedic Surgery, ${ }^{2}$ Radiology and ${ }^{3}$ Pathology, Mie University Graduate School of Medicine, Mie, Japan
}

Received March 9, 2010; Accepted May 24, 2010

DOI: 10.3892/ol_00000105

\begin{abstract}
Ollier's disease is characterized by the hamartomatous proliferation of cartilage cells, producing masses termed chondromas. A patient presented with Ollier's disease which was found to be associated with diffuse gliomas. Investigating this disease is crucial as there is a high risk of sarcomatous transformation of the skeletal lesions as well as an increased risk of developing extra-osseous malignancies.
\end{abstract}

\section{Introduction}

Ollier's disease is characterized by the hamartomatous proliferation of cartilage cells, producing masses termed chondromas. A patient presented with Ollier's disease which was found to be associated with diffuse gliomas. Clinicians should be alert to the possibility of brain tumors during the follow-up of patients with Ollier's disease.

\section{Case report}

A 4-year-old male was referred to our hospital with gait impairment. A shortening of the left leg with a length discrepancy of $3.5 \mathrm{~cm}$ was observed. The patient was using a $3.0 \mathrm{~cm}$ shoe insert. Radiographic findings showed multiple osteolytic changes in his left humerus, femur, tibia and calcaneus (Fig. 1). A bone biopsy was performed from the left distal tibia and the pathology report indicated enchondroma. Subcutaneous hemangiomas were not found, and a clinical diagnosis of Ollier's disease was made. As valgus deformity of the left ankle with a $9 \mathrm{~cm}$ shortening of the left limb was evident, the patient underwent epiphysiodesis of the left ankle and tibia lengthening with the Orthofix external fixator at 10 years of age. Nevertheless, an $11 \mathrm{~cm}$ limb length discrepancy developed with the patient's growth, and he underwent additional limb lengthening with an intramedullary nail at the age of $13 . \mathrm{He}$ received an annual follow-up at the outpatient clinic (Fig. 2).

Correspondence to: Dr Akihiko Matsumine, Department of Orthopaedic Surgery, Mie University Graduate School of Medicine, 2-174, Edobashi, Tsu City, Mie 514-8507, Japan

E-mail: matsumin@clin.medic.mie-u.ac.jp

Key words: Ollier's disease, gliomas, enchondromatosis
At the age of 19, he complained of appetite loss and dysarthria. A neurological evaluation showed GCS E3V3M4, pupil inequality, motor aphasia and right hemiparesis. A head computed tomography scan showed a diffuse low-density area in his cerebrum with an enlarged ventricle. On magnetic resonance imaging, T1-weighted images revealed a diffuse area with low signal-intensity in the brain stem and T2-weighted images showed widespread diffuse infiltrated areas in the brain stem, medulla and cortex, suggesting glioma involvement (Fig. 3A and B). Neurosurgical excision of the brain tumor and ventricular drainage were performed due to evidence of advanced brain herniation and a decerebrate state. Histological specimens from the brain showed increased numbers of glial cells with nuclear atypia and without necrosis or vascular proliferation, establishing the diagnosis of World Health Organization (WHO) grade 3 anaplastic astrocytoma (Fig. 4). Postoperative radiotherapy with chemotherapy (temozolamide, $75 \mathrm{mg} / \mathrm{m}^{2}$ ) were administered. The disease currently remains stable according to the findings of clinical and radiological examinations. Family and patient consent was obtained. Additionally, the patient sample was obtained with the approval of the ethics committee of the Mie University Graduate School of Medicine.

\section{Discussion}

In 1899, Ollier described a developmental anomaly termed 'De la dyschondroplasi' which presented with predominantly metaphyseal non-ossifying cartilaginous masses (1). He noted severe asymmetric, bilateral deformities of the extremities. Ollier's disease, also known as multiple enchondromatosis, is regarded as a congenital disease with unknown etiology. This rare disease is characterized by the hamartomatous growth of cartilage cells, producing masses termed chondromas. Chondromas primarily affect long tubular bones, but involvement of the skull and pelvis is uncommon. Radiographs show large radiolucent lesions within the metaphyses (and to a lesser degree in the epiphyses and diaphyses), leading to the bowing and shortening of the affected bones. The disease is considered to be non-hereditary.

Recent studies with long-term follow-ups of patients with Ollier's disease show a $25-30 \%$ risk of sarcomatous transformation of the cartilaginous lesions (2). Patients with Ollier's disease are generally considered to be less prone to extra-osseous disease. However, Schwartz et al reported that 


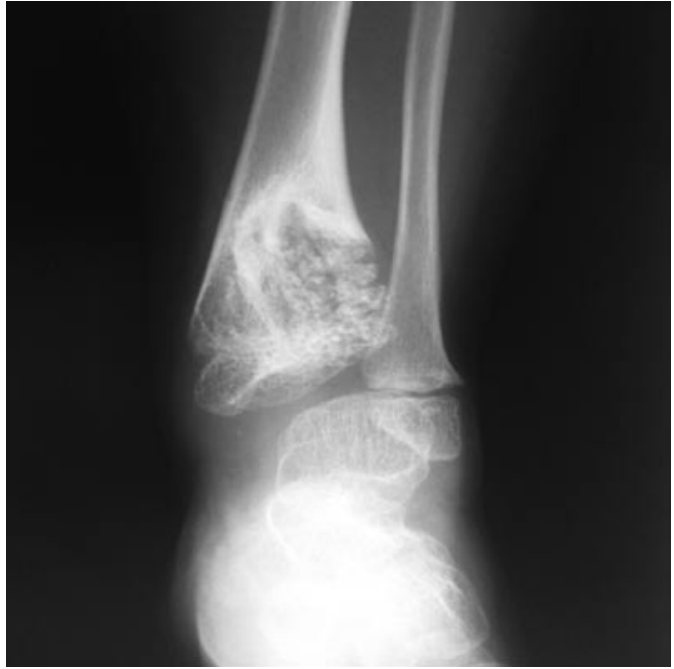

Figure 1. Radiographs of the left distal tibia showing an enchondroma.

\section{A}

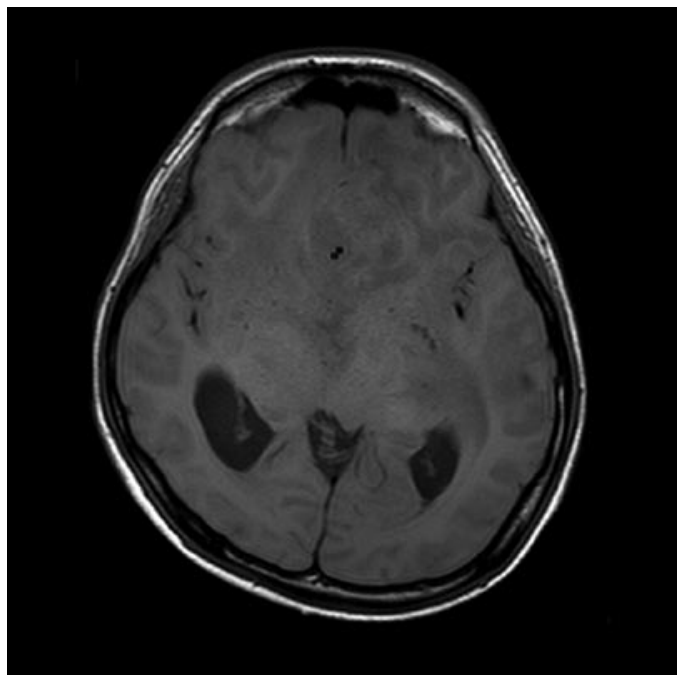

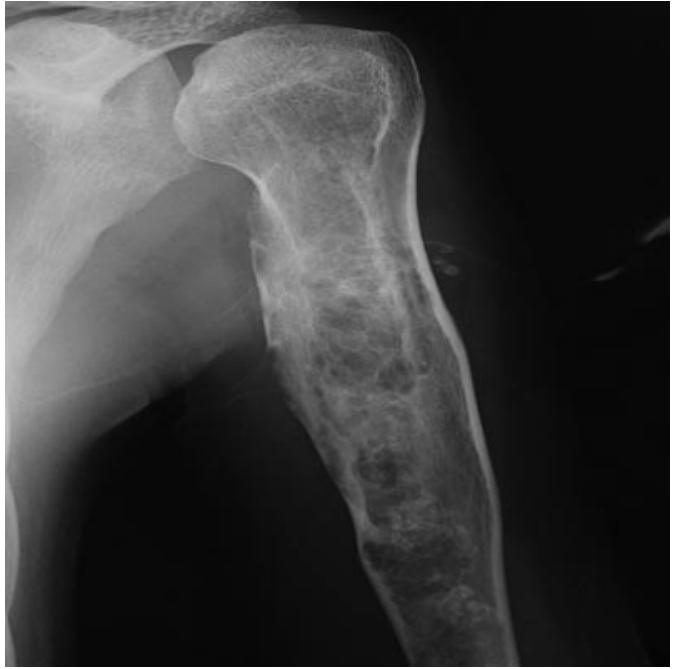

Figure 2. Radiographs of the left humerus showing a large enchondroma involving the metaphysis and proximal diaphysis.

B

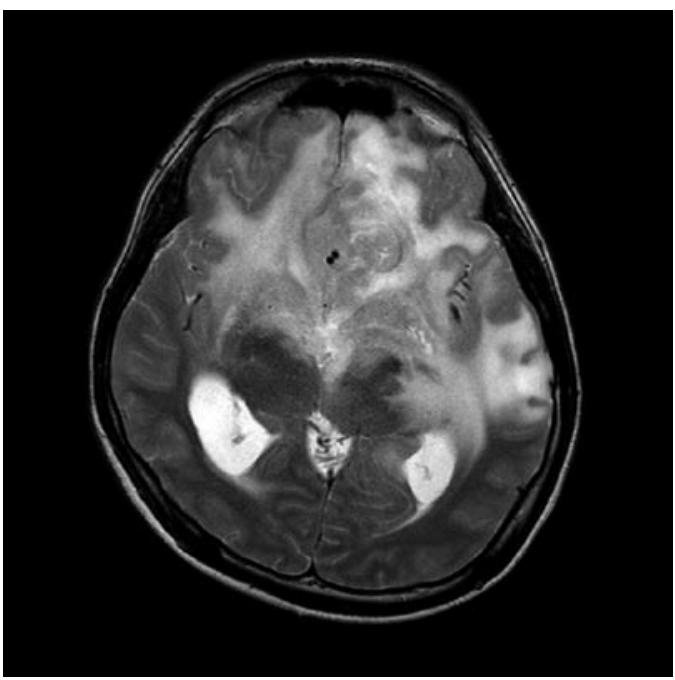

Figure 3. Magnetic resonance image. (A) T1-weighted images showing the diffuse infiltrated area with low signal-intensity. (B) T2-weighted images showing diffuse areas with high signal-intensity in the brain stem, medulla and cortex, suggesting glioma involvement.

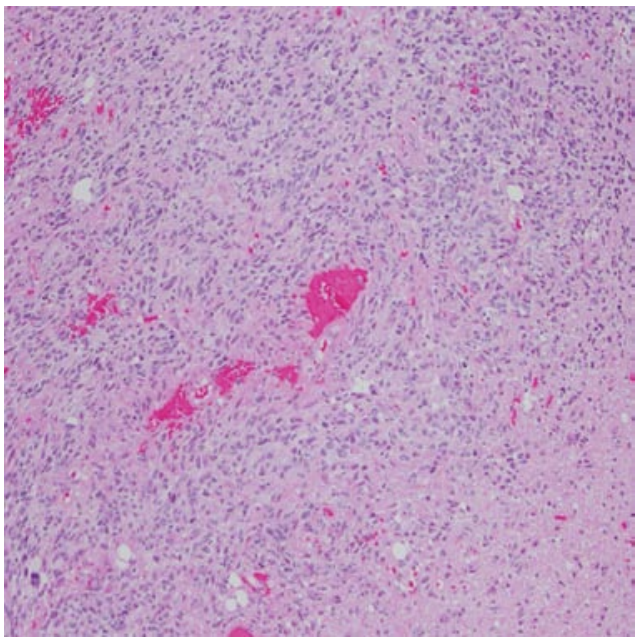

Figure 4. Histological specimen from the brain showing increased numbers of glial cells with nuclear atypia, suggesting WHO grade 3 anaplastic astrocytoma. Mitotic figures are evident in the center (hematoxylin and eosin, $\mathrm{x} 100$ ). out of 37 patients with Ollier's disease, a low-grade chondrosarcoma developed in four, an astrocyoma developed in one and a granulose-cell ovarian tumor developed in another patient (3). Kimmel reported that among 76 cases with Ollier's disease, 29 (35\%) developed non-skeletal tumors. Intracranial neoplasms were noted in 15 patients, including 4 (5\%) with gliomas, 3 with pituitary adenomas, 3 with neuromas and 2 patients with chordomas (4). Therefore, evidence in the literature indicates that gliomas may be relatively frequent in patients with Ollier's disease.

To our knowledge, 14 cases of Ollier's disease associated with gliomas have been reported, including the present case (5-15). The patients were predominantly male and the median age at diagnosis of the glioma was 26.4 years (14-46). No significant differences were found with regard to ethnicity or tumor location. The gliomas were classified as astrocytomas in 10 patients, oligodendroglioma in 2 and glioma with oligodendrocytic components in 1 patient. The standard therapy 
is surgery in combination with radiotherapy and chemotherapy. Outcomes were: 1 continually disease-free patient, 4 no evidence of disease patients, 1 alive with disease and 1 deceased of disease. The results indicated a poor prognosis for patients with glioma and Ollier's disease.

It has been suggested that enchondromas result from abnormalities in signaling pathways controlling proliferation and differentiation of chondrocytes. Consistent with this hypothesis, a functionally deleterious mutation in parathyroid hormone $(\mathrm{PTH})$ /parathyroid hormone-related peptide (PTHrP) type 1 receptor (PTHR1)(R150C) was identified in enchondromas in 2 out of 6 unrelated patients with enchondromatosis (16). PTHR1 interacts with the parathyroid hormone and PTHrP and appears to play a local role in the regulation of chondrocyte differentiation and enchondral ossification (17). On the other hand, PTHrP was found to be expressed in astrocytic tumors (18) and to regulate glioma-associated oncogene transcriptional activation (18). Therefore, there may be a common pathogenesis concerning the $\mathrm{PTH} / \mathrm{PTHrP}$ receptor and ligand with regard to enchondromatosis and glioma. Further research is required to understand the pathophysiology of this rare disease.

The importance of this disease lies in the high risk of sarcomatous transformation of the skeletal lesions as well as in the increased risk of developing extra-osseous malignancies $(19,20)$. Only a limited number of studies address systematic screening for early diagnosis. However, early diagnosis of a brain tumor would improve the prognosis of patients. Since the signs and symptoms of a brain tumor may initially be vague, a neurological examination and magnetic resonance imaging, if necessary, should be conducted during the follow-up period.

\section{References}

1. Shapiro F: Ollier's Disease. An assessment of angular deformity, shortening, and pathological fracture in twenty-one patients. J Bone Joint Surg Am 64: 95-103, 1982.

2. Herbert S and Schwartz MD (eds): AAOS Orthopedic Knowledge Update Musculoskeletal Tumors 2: 108-113, 2007.

3. Schwartz HS, Zimmerman NB, Simon MA, Wroble RR, Millar EA and Bonfiglio M: The malignant potential of enchondromatosis. J Bone Joint Surg Am 69: 269-274, 1987.
4. Kimmel DW, Cheng TM and Mokri B: Primary intracranial neoplasms in Ollier's disease. J Neurooncol 39: 162, 1998.

5. Walid MS and Troup EC: Cerebellar anaplastic astrocytoma in a teenager with Ollier Disease. J Neurooncol 89: 59-62, 2008.

6. Mahafza WS: Multiple enchondromatosis Ollier's disease with two primary brain tumors. Saudi Med J 25: 1261-1263, 2004.

7. Van Nielen KM and de Jong BM: A case of Ollier's disease associated with two intracerebral low-grade gliomas. Clin Neurol Neurosurg 11: 106-110, 1999

8. Frappaz D, Ricci AC, Kohler R, Bret P and Mottolese C: Diffuse brain stem tumor in an adolescent with multiple enchondromatosis (Ollier's disease). Childs Nerv Syst 15: 222-225, 1999.

9. Balcer LJ, Galetta SL, Cornblath WT and Liu GT: Neuroophthalmologic manifestations of Maffucci's syndrome and Ollier's disease. J Neuroophthalmol 19: 62-66, 1999.

10. Hofman S, Heeg M, Klein JP and Krikke AP: Simultaneous occurrence of a supra- and an infratentorial glioma in a patient with Ollier's disease: more evidence for non-mesodermal tumor predisposition in multiple enchondromatosis. Skeletal Radiol 27: 688-691, 1998.

11. Chang S and Prados MD: Identical twins with Ollier's disease and intracranial gliomas: case report. Neurosurgery 34: 903-906, 1994.

12. Bendel CJ and Gelmers HJ: Multiple enchondromatosis (Ollier's disease) complicated by malignant astrocytoma. Eur J Radiol 12: 135-137, 1991.

13. Patt S, Weigel K and Mayer HM: A case of dyschondroplasia associated with brain stem glioma: diagnosis by stereotactic biopsy. Neurosurgery 27: 487-491, 1990.

14. Mellon CD, Carter JE and Owen DB: Ollier's disease and Maffucci's syndrome: distinct entities or a continuum. Case report: enchondromatosis complicated by an intracranial glioma. J Neurol 235: 376-378, 1988.

15. Rawlings CE III, Bullard DE, Burger PC and Friedman AH: A case of Ollier's disease associated with two intracranial gliomas. Neurosurgery 21: 400-403, 1987.

16. Hopyan S, Gokgoz N, Poon R, et al: A mutant PTH/PTHrP type 1 receptor in enchondromatosis. Nat Genet 30: 306-310, 2002.

17. Couvineau A, Wouters V, Bertrant G, et al: PTHR1 mutations associated wirh Ollier disease result in receptor loss of function. Hum Mol Genet 17: 2766-2775, 2008.

18. Alman BA and Wunder JS: Parathyroid hormone-related protein regulates glioma-associated oncogene transcriptional activation: lessons learned from bone development and cartilage neoplasia. Ann NY Acad Sci 1144: 35-41, 2008.

19. Sato K, Hayashi M, Katsumura H, Ishii $\mathrm{H}$ and Kubota T: A case of Maffucci's syndrome with brain-stem tumor. No To Shinkei 41: 631-634, 1989

20. Goto H, Ito Y, Hirayama A, Sakamoto $\mathrm{T}$ and Kowada M: Maffucci's syndrome associated with primary brain tumor: report of a case. No Shinkei Geka 15: 971-975, 1987. 